\title{
Authentizitätsnormen: \\ DGS-Hochsprachlichkeit neu konzeptualisiert
}

\author{
Hanna Jaeger (Leipzig)
}

\begin{abstract}
Both in the academic literature as well in everyday life, the concepts "authenticity" and "norm" are used, but seldom explicitly defined. What is more, at first sight they might not even be regarded as linked. Synoptically considering the findings of three studies on various aspects of authenticity in relation to German Sign Language (Deutsche Gebärdensprache, DGS), the paper explores how both concepts are connected. It will be shown that there are a number of linguistic and para-linguistic features that are perceived as indexical of authentic signing by deaf DGS signers. Drawing on the conceptual distinction between "standard language" (Standardsprache) and "high language" (Hochsprache) introduced by Gloy (1993), it will be argued that whilst these DGS features can be ascribed normative character corresponding to "high language", these are not to be confused with "standard DGS". Apart from newly introducing the concept of "norms of authenticity" (Authentizitätsnormen) in DGS, the paper offers new perspectives in that it will be demonstrated on the basis of sign language data that "standard language" and "high language" may - but in no way necessarily have to - correspond to the same linguistic realizations.
\end{abstract}

\section{$1 \quad$ Einleitung}

Ob der Lieblingsitaliener an der Ecke, der seinen Kunden ,,authentische kulinarische Genüsse“ verspricht, oder die Studienreise, in dessen Rahmen nicht-einheimischen Touristen die lokale Lebensart auf „besonders authentische Weise“ nahegebracht werden soll - ohne Zweifel gehört „Authentizität" zu diesen Begriffen, welche im Alltag zwar regelmäßig verwendet, jedoch so gut wie nie explizit definiert werden. Auch im Zusammenhang wissenschaftlicher Auseinandersetzungen mit Sprachen und Sprechergemeinschaften spielt Authentizität eine große Rolle, nicht nur, wenn es darum geht zu klären, wem man eine linguistische Vorbildfunktion zuschreiben möchte (Buendgens-Kosten 2013). Insbesondere in soziolinguistischen Untersuchungen, welche sich seit Mitte des 20. Jahrhunderts mit „echter Sprache“ bzw. „echtem Sprachverhalten" beschäftigen, ist die Bestrebung, möglichst authentisches Datenmaterial zu analysieren, spätestens seit Labovs Einführung des soziolinguistischen Interviews (Labov 1972) nicht mehr wegzudenken.

Ähnlich vertraut ist das Thema „Normen“. Dass diese nicht nur in der wissenschaftlichen Praxis, sondern auch in der alltäglichen Sprachverwendung einzuhalten sind, gehört zu Alltagserfahrungen, die selten expliziert thematisiert werden. Ob es dabei um die Einhaltung von expliziten Vorschriften i. S. der Befolgung von Prä- und Proskriptionen (Lange 2008: 40) geht, oder 
um das Bespielen verinnerlichter kommunikativer Muster i. S. v. „situationsadäquate[m] sprachliche[m] Handeln“ (Schmitt, 2001: 441) - Normen prägen das soziale Miteinander. Sie werden verstanden, befolgt oder auch missachtet, ohne dass das Konzept an sich für seine Wirksamkeit nach einer Klärung verlangt.

Die folgenden Ausführungen befassen sich mit den Konzepten „Authentizität“ und „Norm“. Neben einer kurzen Betrachtung theoretischer Aspekte soll geklärt werden, wie diese Konzepte zusammenhängen, indem in Bezug auf Sprache bzw. konkrete sprachliche Realisierungen Schnittmengen zwischen diesen beiden Konzepten beleuchtet werden. Folgende zwei Fragen spielen dabei eine besondere Rolle: Gibt es sprachliche Mittel, die als authentisch wahrgenommen werden? Und inwiefern haben Authentizität indizierende Mittel normativen Charakter?

Während sich bisherige Arbeiten vornehmlich mit lautsprachlichem Material befassen, basiert die vorliegende Arbeit auf Ergebnissen, welche in empirischen Studien zur Deutschen Gebärdensprache (DGS) gewonnen wurden. Laut Schätzungen des Deutschen Gehörlosenbundes leben in Deutschland ca. 80.000 gehörlose Menschen. Viele dieser Personen sehen sich selbst als Teil einer Sprachgemeinschaft, welche sich u. a. durch den alltäglichen Gebrauch der DGS im sozialen Miteinander auszeichnet. ${ }^{1}$ Eine Besonderheit der DGS-Gemeinschaft besteht darin, dass die überwiegende Mehrzahl gehörloser Kinder in hörende Familien hineingeboren werden (Mitchell/Karchmer 2004) . Ganz praktisch bedeutet dies, dass ein Großteil der Sprachgemeinschaft DGS nicht als Erstsprache durch ihre Eltern und/oder andere Familienmitglieder im häuslichen Umfeld erlernt. Vielfach werden einschlägige Gebärdensprachkompetenzen erst im Laufe der Kindheit z. B. durch Freundschaften mit Kindern gehörloser Eltern und/oder im Rahmen verschiedener Förderangebote erworben.

In den folgenden Abschnitten wird zunächst erläutert, wie die Konzepte „Norm“ und „Authentizität" theoretisch und konzeptionell zu verorten sind und anschließend skizziert, wie diese Begriffe in der Literatur im Zusammenhang mit Gebärdensprachen verwendet werden. Anschließend werden Authentizität indizierende Mittel in der DGS näher untersucht, bevor ihr normativer Charakter diskutiert wird.

\section{Normen und Authentizität: theoretische Überlegungen}

\subsection{Normen: Sprachliches Handeln im Spannungsfeld von Gebrauch, Erwartung und Bewertung}

Im weitesten Sinne sind Normen als „Orientierungshilfen“ zu verstehen, „nach denen der Mensch sein Verhalten ausrichtet“ (Dittmar 1997: 163). Für ihre Existenz ist es nicht zwangsweise notwendig, dass sie in irgendeiner Form festgeschrieben, i. e. kodifiziert, sind, da sie zum Teil als unbewusstes Weltwissen tradiert werden und entsprechend im sozialen Miteinander ihre Wirksamkeit entfalten. Das heißt, ,'Sprachnormen' sind also nicht etwa sprachlich fixierte Normen überhaupt, i. e. Normformulierungen, sondern soziale Normen, die - gleichgültig, ob formuliert oder nicht formuliert - den Umfang, die Auswahl, den spezifischen Gebrauch von Sprachmitteln in irgendeiner Hinsicht als verbindlich festlegen." (Gloy 1975: 61; zitiert nach

\footnotetext{
${ }^{1}$ Berücksichtigt man, dass auch hörende Eltern und Geschwister gehörloser Kinder, hörende Kinder gehörloser Eltern, Gebärdensprachdolmetscher, Sozialarbeiter etc. regelmäßig in DGS kommunizieren, so ist die Anzahl der DGS-Nutzenden höher als die Zahl gehörloser Personen.
} 
Tauber 1993: 197). Dass Normen wirksam sind, ist zum einen daran erkennbar, dass ihre Befolgung empirisch nachvollziehbar und dokumentierbar ist. Andererseits lässt sich die Existenz von Normen auch dann erkennen, dass deren Verletzung Sanktionen nach sich ziehen kann. Geht man davon aus, dass sprachliches Handeln ein Ausdruck sozialer Interaktion ist, dann lassen sich Sprachnormen, welche sprachliches Handeln regeln, als „spezieller Fall sozialer Normen“ (Hartung 1977: 11) einordnen.

In der Literatur lässt sich die Auseinandersetzung mit Sprachnormen aus unterschiedlichen Perspektiven nachvollziehen. Das konkrete Verständnis des verwendeten Normbegriffes orientiert sich dabei maßgeblich an der wissenschaftlichen Disziplin, im Kontext derer diese Auseinandersetzungen stattfinden. Dittmar (1997: 164) argumentiert, dass sich in der Linguistik im Grunde genommen zwei Normkonzepte etabliert haben. Auf der einen Seite gibt es den etwas enger gefassten Ansatz, in welchem Norm als „die Regel“ verstanden wird. Das heißt, aus „funktional-systemhaften Aspekten“ wird untersucht, „,was grammatisch korrekt und semantisch interpretierbar“ ist (ibid.). Dem gegenüberstehend sieht der Autor den etwas weiter gefassten, ,koexistierende[n] Ansatz“, in dessen Rahmen davon ausgegangen wird, dass es nicht die eine Norm gibt, sondern dass mehrere Normen nebeneinander existieren. Um diese zu verstehen, müssen soziale und situative Faktoren berücksichtigt werden (ibid.). Während die erstgenannte ,einer im strengen Sinne linguistischen“ Perspektive entspricht, ist die zweitgenannte „einer im weiteren Sinne soziolinguistischen“ Sichtweise zuzuschreiben (Dittmar 1997: 165).

Brommer (2015: 122) unterscheidet zwischen einem ,soziolinguistische(n) Verständnis von ,Norm' als ,Erwartung' und ,Verpflichtung' und [... dem ...] pragmatische(n) Verständnis von ,Norm' als ,Gebrauchsnorm'“. Verweisend u. a. auf Arbeiten von Gloy (1975; 1995; 2004) argumentiert die Autorin, dass erstere Sicht davon ausgeht, dass die „Erwartung einer Regelmäßigkeit"“ (2015: 122) im Hinblick auf die Okkurrenz oder Absenz eines sprachlichen Phänomens in einem bestimmten Kontext wesentlicher Bestandteil von Normen ist. In welchem Ausmaß sich diese Regelmäßigkeit darstellt, ist dabei für die Existenz der Norm unerheblich, da diese auch dann existiert,

„wenn die Regelmäßigkeit nicht eingehalten wird. Das Unregelmäßige wird dann sanktioniert, kritisiert oder korrigiert oder als eine Ausnahme akzeptiert. Die Norm ist SollZustand, sie besteht also unabhängig vom tatsächlichen Verhalten/Handeln und ist damit mehr als eine bloße Erwartung einer Regelmäßigkeit, die bei häufigem Nichterfüllen der Regelmäßigkeit aufgehoben würde“

(Brommer 2015: 122).

Dieser soziolinguistisch orientierten Perspektive steht das pragmatische Normverständnis gegenüber, welches sich im Wesentlichen dadurch auszeichnet, dass Norm für all das steht, was üblicherweise im alltäglichen Sprachgebrauch vorkommt. Setzt man also Norm mit dem IstZustand gleich, dann enthält diese ,all das, was in der einer funktionellen Sprache entsprechenden Rede traditionell, allgemein und beständig [...] ist, nämlich alles, was man ,so und nicht anders' sagt (und versteht)“ (Coseriu 1988: 297 nach Brommer 2015: 122). So gesehen ließe sich Norm „gleichsetzen mit ,Usus'“, was wiederum dazu führt, dass das, „was als Norm gilt, 
empirisch identifiziert werden“" kann (Brommer 2015: 122). ${ }^{2}$ In gewisser Hinsicht hängen natürlich die Aspekte „Erwartung“ und „Gebrauch“voneinander ab. Was regelmäßig auftritt, wird irgendwann auch erwartet. Der Unterschied in der von Brommer (2015) skizzierten Unterscheidung zwischen soziolinguistischen und pragmatischen Perspektiven, liegt in dem Fokus der Betrachtung. Konzentriert sich erstere Perspektive auf die Einstellung des Produzenten/Rezipienten, rückt letztere primär das sprachliche Phänomen in den Fokus. Beide Sichtweisen beinhalten jedoch jeweils beide Facetten.

Eingebettet in Diskurse der Sprachkritik und aufbauend auf Techtmeier (1977) argumentiert Fix (1995: 67), dass verschiedene Perspektiven und Zugänge zu sprachlichen Normen letztlich im Begriff „Angemessenheit“ einen gemeinsamen Nenner finden. Zunächst zeigt sie auf, dass sich sprachlich-kommunikative Normen in vier verschiedene Subkategorien differenzieren lassen. Die „instrumentale Norm“ bezieht sich auf das einer Sprache zugrunde liegende System, wie beispielsweise ihre Grammatik und Semantik. Die Kategorie „situative Normen“, bezieht sich primär auf Faktoren, welche die Situation näher gestalten. Unter der dritten Kategorie, „ästhetische Normen“, lassen sich Ansprüche an die Klarheit, Gewähltheit und Elaboriertheit eines Textes subsummieren. Der vierten Kategorie, ,,parasprachliche Normen“, lassen sich Normen zuordnen, welche Aspekte außersprachlicher Codes in den Vordergrund rücken. Die Angemessenheit eines Textes wird also unter Rückgriff auf kulturelle Bedingungen und Traditionen geprüft, indem die Frage gestellt wird, ob dieser als kulturell adäquat oder inadäquat einzuordnen ist (Fix 1995: 67). Nach dem von Fix (1995: 67) entworfenen Model müssen normative Betrachtungen von Texten (in welcher Form auch immer sie realisiert werden) auf mehreren Ebenen vollzogen werden. Das heißt, die Bewertung sprachlichen Handelns orientiert sich nicht nur an linguistischen Aspekten (instrumentale und parasprachliche Normen), sondern ist immer auch an kulturelle Bedingungen (ästhetische und situative Normen) geknüpft.

\subsection{Standard: eine prototypische Sprachnorm}

Werden in der Literatur sprachliche Normen thematisiert, so geschieht dies häufig im Zusammenhang mit Standardvarietäten. Dies ist insofern nicht verwunderlich, als dass es sich hierbei um die sprachliche Varietät handelt, welche - im Vergleich zu anderen Varietäten - über das höchste Maß an kodifizierten Normen verfügt, und deren Missachtung i. d. R. sanktioniert werden. Interessanterweise sind die Konzepte „Norm“ und „Standard“ dabei häufig so eng miteinander verwoben, dass die Begriffe gar nicht so selten inhaltlich synonym verwendet werden, oder, wie Ammon (2005) es treffend formuliert: „Der Standard wird auch oft mit der Norm der Sprache schlechthin gleichgesetzt." Gelegentlich werden beide Termini gar in einem Kompositum (,Standardnorm“) zusammengeführt (Eichinger 2005).

Ungeachtet seiner Verbreitung gibt es keine einheitliche Definition des Konzepts „Standardvarietät". Je nach Autor werden stattdessen unterschiedliche Aspekte in den Vordergrund gerückt. Bei Ammon (2005) sind es bspw. Kodifiziertheit, Gegenstand förmlichen Sprachunterrichts und der amtliche Status. Bußmann (2008) dagegen betont seine Überregionalität und Assoziation mit sozialer Mittel-und Oberschicht. Während Behrend (2005) noch diskutiert, inwiefern

\footnotetext{
${ }^{2}$ Eine Möglichkeit, regelmäßig wiederholende Sprachgebrauchsmuster zu identifizieren und diese in Form von Regeln zu übersetzen und als Sprachgebrauchsnormen zu interpretieren, bieten bspw. korpuslinguistische Arbeiten (Brommer 2015: 122).
} 
es nicht nur nationale, sondern auch regionale Gebrauchsstandards gibt, setzen sich Schneider und Albert (2013) mit der Frage auseinander, welche Bedeutung in diesem Zusammenhang Aspekten wie Medialität zugesprochen werden muss. ${ }^{3}$

Werden in der Literatur, wie bereits angemerkt, die Termini Norm und Standard oft synonym verwendet, lässt sich ebenso beobachten, dass mit den Begriffen Hochsprache und Standardsprache ähnlich verfahren wird. Das heißt, auch sie werden scheinbar austauschbar zur näheren Bestimmung ein und desselben Gegenstandes verwendet (Löffler 2005). Gloy (1993: 55) kritisiert diese Praxis und schlägt darum vor, nicht nur eine terminologische, sondern tatsächlich eine konzeptionelle Trennung vorzunehmen. Der Autor betont, dass es sich hier um zwei verschiedene Varietäten handelt, welche ,sich zwar (im Deutschen) auf die Sprachform der höheren Sozialschichten“ beziehen, dabei jedoch sehr unterschiedliche Aspekte in den Vordergrund rücken. Während ,>Standardsprache $<$ [...] den offiziell kodifizierten Normkomplex mit weitestgehender Überdachungsfunktion“ meint, steht der Begriff ,, $>$ Hochsprache $<$ [für] eine Prestige-Varietät, deren Beschaffenheit und Wert sich nicht an Kodifikationen, auch nicht an Überregionalität bemißt, sondern allein daran, wer diese Varietät spricht.“ (Gloy 1993: 55, Hervorhebung hinzugefügt). Der Autor differenziert also zwischen der Bezugsgröße, welche für das Verständnis des jeweiligen Begriffes entscheidend ist. Bei „Standardsprache“ ist es das linguistische Repertoire (,was“), bei „Hochsprache“ ist es die sich dieses Repertoires bedienende Population (,wer").

An dieser Stelle muss hervorgehoben werden, dass sich Gloy explizit auf die deutsche Lautsprache bezieht. Auch andere Autoren beziehen sich in ihren Ausführungen primär auf die soziolinguistische Situation von Lautsprachen. Dies ist nicht ungewöhnlich, zeigt jedoch, dass die Entwicklung soziolinguistischer Konzepte bisher eher selten unter Berücksichtigung gebärdensprachlicher Aspekte erfolgt.

Der vorliegende Aufsatz setzt sich explizit mit Aspekten sprachlicher Normen in DGS auseinander. Ausgehend von einem Normkonzept, das als verbindliches sprachliches Handeln im Spannungsfeld von Erwartung, Gebrauch und Bewertung definiert wird, wird der Frage nachgegangen, welche sprachlichen und parasprachlichen Realisierungen normativen Charakter aufweisen. In den Fokus rücken dabei solche Mittel, welche mit authentischen Gebärdensprachgebrauch assoziiert werden. Bevor dies anhand empirischen Datenmaterials betrachtet wird, soll auf das Konzept „Authentizität“ eingegangen werden. Zunächst werden theoretische Positionen beleuchtet, welche Authentizität als philosophisches Konstrukt begreifen. Anschließend geht es um eine Annäherung an den Begriff aus Sicht der Angewandten Linguistik. Hier wird u. a. der Frage nachgegangen, welche praktische Relevanz dem Konzept im Fremdsprachunterricht zukommt.

\subsection{Authentizität: Theoretische Überlegungen und angewandte Kontexte}

„Authentizität“" ist zweifelsohne einer jener Begriffe, welche im Alltag zwar häufig Verwendung finden, gleichzeitig jedoch selten definiert werden. In der Regel wird der Begriff

\footnotetext{
3 Inwiefern es sich bei Standard um ein „,ideologisches Konstrukt“ handelt (Richter 2005: 136), oder ob sich eine solche sprachliche Ausprägung tatsächlich empirisch beschreiben lässt, ist Gegenstand von Diskussionen (cf. dazu Bex/Watts 1999).
} 
verwendet, wenn es darum geht, die Genuinität oder Echtheit einer Sache, einer Person oder eines Events zu betonen. Häufig findet der Begriff auch Anwendung, wenn explizit Bezüge zur Historizität hervorgehoben werden sollen. „In its widest sense, , authenticity“ is related to notions of ,realness“ or ,trueness to origin“" (Buendgens-Kosten 2014: 457). Dass das Konzept „Authentizität" irgendetwas mit Echtheit zu tun hat, sich gleichzeitig jedoch schwer auf den Punkt bringen lässt, betont auch Akande (2014: 270), indem er, bezugnehmend auf Rose (2008), feststellt: "Authenticity, or keepin'it real, is a complex concept which does not lend itself to a fixed definition". Angesichts der Abwesenheit einer festen Definition, sollen kurz einige Perspektiven skizziert werden, aus denen das Konzept betrachtet werden kann.

Lacoste et al. (2014: 2) begreifen „Authentizität“ in erster Linie als ein philosophisches Konstrukt und unterscheiden in ihren Ausführungen zwischen statischen, relationalen, dynamischen, attributiven und interaktionalen Perspektiven. Von einem statischen (oder auch essentialistischen) Standpunkt aus betrachtet, rückt die Auffassung in den Vordergrund, dass Authentizität in besonderer Weise als eine intrinsische Eigenschaft eines Objektes darstellt, unabhängig davon, ob es sich dabei um ein belebtes oder unbelebtes Objekt handelt. Diese ontologische Perspektive auf Authentizität lässt sich beispielsweise in der expressiven Qualität eines Artefaktes identifizieren, aber auch in dem Wesenszustand einer Persönlichkeit (cf. Heidegger 1927). Die relationale Sichtweise, wie sie u. a. bei Sartre (1945) zu finden ist, konzeptualisiert Authentizität als etwas, was zwar in der Art, wie es sich manifestiert, festgeschrieben ist, gleichzeitig jedoch seine Bedeutung aus der Beziehung zu etwas oder jemandem erhält. Diese relationale Sichtweise zeigt sich beispielsweise in Standards oder Werten. Authentizität lässt sich ebenso als dynamischer Prozess begreifen, und zwar im Sinne eines Ergebnisses eines Authentifizierungsprozesses. Aus dieser Sichtweise ist Authentizität messbar, beispielweise durch Validierung des Alters eines Gegenstandes oder Materials. Die Auffassung, Authentizität liege im Auge des Betrachters, basiere also darauf, inwieweit ein Publikum bei einem entsprechenden Event entsprechend emotional bewegt ist, beschreiben Lacoste et al. (2014) als „attributionale“ Perspektive. Im Kontrast dazu erfassen sie unter der ,interaktionalen“ Perspektive diejenige Sichtweise, welche Authentizität als das kreative Ergebnis eines komplexen Zusammenspiels zwischen einem Produzenten, einem Produkt und Rezipienten begreift (cf. Gadamer 1960). Die aufgeführten Sichtweisen zeigen, dass das Verständnis des Konzepts Authentizität im Wesentlichen von der Positionierung des Betrachtenden abhängt, wobei sich das Spektrum möglicher Verortungen zwischen den Extremen „statisch“ und „dynamisch“ erstreckt.

Während der Ursprung des Verständnisses von „Authentizität“ als ideologisches Konstrukt, welches sowohl die Sprachverwendung von Sprechern als auch die wissenschaftliche Analyse eben dieser Sprachpraxis untermauert (Eckert 2003: 392), nicht auszumachen ist, wird das Thema „Authentizität“ in der soziolinguistischen Literatur seit Anfang der 2000er kritisch hinterfragt (Eckert 2003; Coupland 2003; Bucholtz 2003) und weiterentwickelt (Pietikäinen et al. 2016). Eckert (2003) argumentiert, dass das im Strukturalismus verankerte Verständnis von sprachlichen Varietäten als statische, fixierte Einheiten oftmals auf „authentische Sprache“ übertragen wird. Erfolgt darüber hinaus eine Verknüpfung von konkreten Merkmalen (z. B. Verhalten, Einstellungen) mit bestimmten Gruppen, mag dies aus essentialistischer Sicht stimmig sein. Bucholtz (2003) hält jedoch dagegen, dass auch das, was in manchen Kontexten als 
„authentische Sprache“ gelten mag, niemals statisch ist, sondern in Abhängigkeit wechselnder kontextueller Faktoren immer wieder neu ausgehandelt werden muss.

Indem er behauptet: ,language in itself can never be said to be authentic, but there are ways in which language can be and has been associated with authenticity", vertritt Coupland (2014:21) ein Verständnis von Authentizität als interaktional konstruiertes Konzept. Dieses ist vergleichbar mit Spitzmüllers (2013: 404), wenn er argumentiert, dass Authentizität nicht so sehr als ein ontologisches Charakteristikum bestimmter Kommunikationsformen, sondern viel mehr als „soziale(s) und (meta-)kommunikative(s) Konstrukt (...) mit spezifischer sozialer Bedeutung“ zu verstehen ist. Mit anderen Worten, Authentizität ,ist sozialer Sinn [...] ein sozialer Wert, der durch [...] Authentifizierungsprozesse [...] kommunikativ konstruiert und interaktiv verhandelt wird“" (Spitzmüller 2013: 410f., Hervorhebung im Original).

Im Gegensatz zu eher theoretisch motivierten Überlegungen zum Thema „Authentizität“ im Rahmen philosophischer Betrachtungen stehen in Auseinandersetzungen mit dem Konzept „Authentizität“ im Bereich des Fremdsprachen Lehrens und Lernens eher angewandte Perspektiven im Vordergrund. So argumentiert Gilmore (2007), dass seit den 1970ern das Thema Authentizität sehr an Bedeutung im ELT-Bereich gewonnen hat, da sich zunehmend die Auffassung verbreitete, dass erfolgreiches Fremdsprachenlernen wesentlich mehr umfassen muss, als sprachlich-strukturelle Konstruktionen zu beherrschen.

Im Zuge dieser Entwicklungen galt es zu klären, was Authentizität im Zusammenhang mit strukturellen und performativen Aspekten verschiedener Sprachen und Texte bedeutet, wie man authentisches nicht-sprachliches Verhalten definiert und was das Konzept beinhaltet, wenn es darum geht, authentische Situationen zu definieren. Nicht zuletzt sind es auch die Fragen, was genau unter einem authentischen Sprachnutzer zu verstehen sei (Coupland 2007: 180), oder was die Rolle eines Muttersprachlers als die personifizierte Quelle sprachlicher Authentizität definiert (Lowe/Pinner 2016), welche das Thema für den Fremdsprachbereich relevant macht. Insbesondere der der Verweis auf Sprachverwender, welchen auf Grundlage ihrer Spracherwerbsbiographie authentischer Status zugesprochen, zeigt auf, dass in sprachbezogenen Diskursen das Konzept Authentizität ,den Mittelpunkt der hitzigsten sprachideologischen Kämpfe darstell[t]““ (Spitzmüller 2013: 429).

In Diskursen über Minderheitensprachen tritt diese sprachideologische Dimension von Authentizität hervor. So schildert Matras (2015: 308f.) bspw. Reaktionen auf die Veröffentlichung eines Wörterbuchs (Roma-Serbisch) im August 2011 durch den Serbisch-Roma Aktivisten und Autoren Bajram Haliti. Auffallend in diesem Zusammenhang sei, dass "[a]uthenticity, understood to be a claim to Romani ancestry, is flagged as an indication of the credibility and reliability of an academic enterprise". Diese Aussage mag in zweierlei Hinsicht verblüffen. Zum einen überrascht die Tatsache, dass Haliti in diesem speziellen Bereich authentischer Status qua Herkunft zugesprochen wird, i. e., dass er seine Position als authentischer Vertreter der Roma Population quasi als Geburtsrecht ererbt hat. Nicht weniger überraschend erscheint der zweite Aspekt, nämlich dass dieser Authentizitätszuspruch qua Geburt auf die Wahrnehmung der Qualität seines wissenschaftlichen Schaffens übertragen wird. Mit anderen Worten: einer Person wird aufgrund biographischer Faktoren (Geburt) der Status ,authentisch“ zugesprochen. Gleichzeitig wird dieser Authentizitätszuspruch als Garant für wissenschaftliche Glaubwürdigkeit und Seriösität interpretiert. Der in diesem Beispiel sichtbare ideologische Unterbau von 
Authentizitätskonzepten wird im Zusammenhang mit Minderheitensprachen auch von anderen Autoren kritisch betrachtet, insbesondere dann, wenn die Sprachverwendung von Muttersprachlern mit ,authenticity and non-native forms with artificiality and hybridity“ (O’Rourke/Ramallo 2013: 290) gleichgesetzt wird.

\subsection{Normen und Authentizität im Kontext von Gebärdensprachen}

Ist in der Literatur von gebärdensprachlichen Normen die Rede, so geschieht dies vorrangig im Zusammenhang mit Spracherwerb oder Standardisierung. Im ersten Fall geht es darum zu klären, welche Kriterien sich als Bemessungsgrundlagen im Hinblick auf die Bewertung von Gebärdensprachkompetenzen (Woll 2013) eignen. Im zweiten Fall werden u. a. Fragen bezüglich Herausforderungen und Umsetzung politisch geforderter Standardisierungsbemühungen (Schermer 2012) erörtert.

Ungeachtet der Tatsache, dass es bisher keine kodifizierte Standardvarietät in der Deutschen Gebärdensprache (DGS) gibt, lässt sich jedoch auch hier ein Verständnis von Standard als Inbegriff einer sprachlichen Norm identifizieren. Aus Sicht gehörloser Gebärdensprachverwender ist der Terminus und das damit assoziierte Konzept „Standard“ tendenziell negativ besetzt. So argumentiert Eichmann (2009a; 2009b; 2013), dass die Frage scheinbar fehlender Standardvarietäten in Gebärdensprachen in erster Linie sprachpolitische und sprachplanerische Aktivitäten hörender Personen motiviert und folglich das Konzept „Gebärdensprachstandardisierung“ von Gebärdensprachnutzern in Deutschland und Großbritannien häufig als „Hegemonie Hörender" konstruiert wird (cf. Adam 2015).

An anderer Stelle wird diskutiert, inwiefern im Hinblick auf Standardsprachlichkeit in Gebärdensprachen die gleichen Faktoren eine Rolle spielen, wie sie für Lautsprachen als relevant angenommen werden. So hinterfragt Jaeger (2017), ob das von Ammon (2003) für Lautsprachen beschriebene soziale Kräftefeld, welches sprachliche Standards bestimmt, in gleicher Weise in Gebärdensprachgemeinschaften wirksam ist. Während Johnston (2003) noch grundsätzlich die Möglichkeit der Standardisierung von Gebärdensprachen in Abwesenheit einer Gebrauchsschrift in Frage stellt, spekuliert Krentz (2006) darüber, ob sich letztendlich wohl die Kamera als die „Druckerpresse“ der Gebärdensprache herausstellen wird. Das Beispiel der fehlenden Gebrauchsschrift macht deutlich, dass in expliziten Auseinandersetzungen mit Fragen von Standardsprachlichkeit, ähnlich wie in Diskussionen bezüglich Lautsprachen, auch in Gebärdensprach-Kontexten primär linguistische Aspekte im Fokus stehen. Sieht man von Diskussionen bezüglich der Notwendigkeit, Erwünschtheit oder sozialen Bedeutung von Gebärdensprachstandardisierung ab, so geht es vor allem darum zu klären, wie ein gebärdensprachlicher Standard aus linguistischer Perspektive zu beschreiben wäre und weniger darum, welches soziale Prestige (wenn überhaupt) mit so einer Sprachform assoziiert wird. ${ }^{4}$

\footnotetext{
${ }^{4}$ Im Übrigen tritt auch in angewandten Kontexten das Thema Standard-DGS in Erscheinung. So wird im Rahmenlehrplan für die Bundesländer Berlin und Brandenburg für die Jahrgangsstufen 1-10 (2015: 17), ,Funktionale kommunikative Kompetenz“ von Schülerinnen und Schülern im Bereich „Dialogisches Sehverstehen“ auf Stufe (G) dahingehend beschrieben, dass sie ,in authentischen Sehtexten unterschiedlicher Textsorten wichtige Informationen verstehen, wenn in deutlich artikulierter Standard- bzw. unterrichtsbezogener Fachsprache gebärdet wird“. Interessanterweise werden hier Kompetenzen definiert, welche sich an dem Umgang mit einer gebärdensprachlichen Varietät (Standard) orientieren, deren Existenz bisher weder konkret dokumentiert, geschweige denn
} 
Obwohl er selten explizit definiert wird, findet der Begriff „Authentizität“ in der gebärdensprachlinguistischen Literatur immer wieder Verwendung. Insbesondere im Zusammenhang mit der Erhebung von gebärdensprachlichem Datenmaterial geht es häufig um die Abgrenzung zwischen „simulated“ versus ,authentic data“, wobei ,,authentic sings“ als synonym mit ,naturally occuring data“ verstanden wird (Napier/Leeson 2016). Eine ähnliche Unterscheidung wird von Morford/Nicodemus/Wilkinson (2015: 213) vollzogen, wenn sie argumentieren, dass sich ,authentic signs, such as foreign signs“ möglicherweise eher als Stimulusmaterial in einschlägigen Studien eignen, als konstruierte und somit künstliche Gebärdenzeichen. Zwar rücken Langer et al. (2018) die Bedeutung der Verwendung von „Authentic Examples in a Corpus-Based Sign Language Dictionary“ allein schon durch den Titel ihres Aufsatzes in den Fokus. Allerdings vermeiden auch sie es, näher zu definieren, was hier genau mit ,authentisch“ gemeint ist. Ungeachtet der Abwesenheit expliziter Definitionen legt seine Verwendung in den genannten Beispielen nahe, dass diese auf einem Konzeptverständnis basiert, welches Authentizität als intrinsische Eigenschaft in den Vordergrund rückt und somit am essentialistischen Ende des Spektrums zu verorten ist.

Während bis hierher Normen und Authentizität als theoretische Konzepte skizziert worden sind, soll in den folgenden Abschnitten auf der Basis von empirisch generiertem Datenmaterial der Frage nachgegangen werden, ob es gebärdensprachliche Mittel gibt, die von DGS-Nutzern als authentisch wahrgenommen werden. Darüber hinaus soll in der Betrachtung entsprechender Daten ausgelotet werden, inwiefern diesen sprachlichen Realisierungen normativer Charakter zugeschrieben werden kann.

\section{Authentischer Gebärdensprachgebrauch: Empirische Annäherungen}

Während das Thema „Authentizität“ als Gegenstand theoretischer Auseinandersetzungen in der Literatur zu Gebärdensprachen insgesamt eher unterrepräsentiert ist, haben sich Jaeger (2018), Jaeger und Harzer (2019) und Jaeger (2019) mit der Frage auseinandergesetzt, welche sprachlichen Merkmale in der DGS Authentizität indizieren.

\subsection{Datenbasis}

Die Analysen der in den folgenden Abschnitten betrachteten Einzelstudien basieren auf einem,

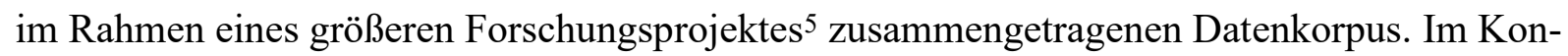
kreten handelt es sich bei den Daten um ein Gesprächskorpus, welches aus zehn FokusgruppenGesprächen mit jeweils vier gehörlosen Personen besteht. Die Gespräche, welche im Herbst 2015 an neun Standorten in Deutschland geführt wurden, wurden von ein und derselben gehörlosen Projektmitarbeiterin moderiert. Die Fokusgruppen wurden in DGS geführt und deckten thematisch eine Reihe unterschiedlicher Bereiche ab. So ging es u. a. um ästhetische Empfindungen (Was ist schöne Gebärdensprache?), soziale Aspekte (Sprachvorbilder), normative Aspekte gebärdensprachlicher Realisierungen (Unterschied zwischen RICHTIG und ECHT), und

\footnotetext{
kodifiziert ist. In gleicher Weise wird auch an keiner Stelle erklärt, was genau unter ,authentischen Sehtexten“ zu verstehen ist.

${ }^{5}$ Das Forschungsprojekt "Normenemergenz in der Deutschen Gebärdensprache (DGS)” wurde von März 2015 bis Februar 2018 an der Universität Leipzig durchgeführt und von der Deutschen Forschungsgemeinschaft (DFG) gefördert [EI 911/2-1].
}

ISSN 1615-3014 
die Frage, welche sprachlichen und para-sprachlichen Merkmale aus Sicht gehörloser DGSNutzer authentisches Sprachverhalten indizieren (Wie sieht „typisch taube“ DGS aus?). Die Fokusgruppen wurden jeweils aus drei verschiedenen Kameraperspektiven aufgezeichnet. Im Anschluss wurde das Datenmaterial für die Analyse aufbereitet, indem die jeweils drei einzelnen Videofiles pro Gespräch in jeweils ein Videofile zusammengeführt wurden. Diese wurden anschließend in der Software ELAN transkribiert und unter qualitativen und quantitativen Gesichtspunkten analysiert.

In den kommenden Abschnitten sollen einschlägige Ergebnisse der genannten Teilstudien (TS_1, TS_2 und TS_3) beleuchtet und anschließend der Zusammenhang zwischen Normen und Authentizität aufgegriffen, herausgearbeitet und diskutiert werden.

\subsection{TS_1: Authentizität - konzeptualisiert als sprachliche Varietät}

In ihren Ausführungen beschäftigt sich Jaeger (2018) mit metasprachlichen Kommentaren gehörloser Gebärdensprachnutzer bezüglich der Frage, an welchen Aspekten erkennbar sei, ob eine gebärdensprachliche Äußerung als authentisch bzw. nicht authentisch wahrgenommen wird. Als theoretischen Ausgangspunkt bedient sich die Autorin der perzeptuellen Perspektive im Sinne der von Niedzielski und Preston (2000) vertretenen Folk Linguistik. Untersuchungen in dieser Tradition zielen u. a. darauf ab, die areale Verteilung sprachlicher Merkmale aus laienlinguistischer Sicht zu erheben (Pearce 2012). Jaeger (2018) greift diesen Ansatz auf, verwendet ihn jedoch auf metaphorischer Ebene, um zu zeigen, dass es eine Reihe von sprachlichen Elementen gibt, welche aus laienlinguistischer Sicht durchaus ,,als relevant in der Konstruktion von (Nicht-) Authentizität als soziales Territorium innerhalb der DGS-Gemeinschaft wahrgenommen werden“ (Jaeger 2018: 76, eigene Übersetzung).

In ihren Ausführungen zeigt die Autorin, dass gehörlose Gebärdensprachnutzer über ein ausgeprägtes Narrativ verfügen, aus dem hervorgeht, an welchen Merkmalen es sich für sie festmacht, ob sie eine DGS-Äußerung als authentisch oder nicht-authentisch wahrnehmen. Im Wesentlichen lassen sich diese Merkmale in drei Kategorien einteilen (siehe Tabelle 1). 


\begin{tabular}{|c|c|}
\hline Kategorie & Merkmale \\
\hline $\begin{array}{l}\text { I: Prosodische und parasprachli- } \\
\text { che Aspekte }\end{array}$ & $\begin{array}{l}\text { Geschwindigkeit, } \\
\text { Natürlichkeit, } \\
\text { Charisma }\end{array}$ \\
\hline II: Strukturelle Aspekte & $\begin{array}{l}\text { lexikalisches Inventar (Gebärdenschatz), } \\
\text { Breite des aktiv genutzten lexikalischen Spektrums, } \\
\text { idiomatische Ausdrücke, } \\
\text { verwendete grammatische Strukturen }\end{array}$ \\
\hline \multirow[t]{2}{*}{ III: Artikulatorische Aspekte } & $\begin{array}{l}\text { IIIa: Mimik (allgemein): } \\
\text { Intensität, } \\
\text { Quantität, } \\
\text { Adäquatheit }\end{array}$ \\
\hline & $\begin{array}{l}\text { IIIb: Mimik (speziell Mund): } \\
\text { Mundgestik } \\
\text { Ablesewörter }\end{array}$ \\
\hline
\end{tabular}

Tabelle 1: Aspekte, die aus laienlinguistischer Perspektive relevant für die Beurteilung ,, authentischer DGS“6 wahrgenommen werden (Jaeger 2018)

Zunächst hält sich die Annahme, dass bestimmte prosodische und parasprachliche Aspekte Authentizität indizieren (siehe Kategorie I). Dazu gehören Geschwindigkeit, Natürlichkeit und Charisma (Jaeger 2018: 90-91). Der Kategorie II zugeordnet sind eher strukturelle Aspekte, wie sie sich beispielsweise auf der Ebene des Lexikons nachweisen lassen, zuzuordnen. So gehen laut Jaeger (2018: 86-89) die Probanden davon aus, dass sich u. a. an der Breite des aktiv genutzten lexikalischen Spektrums, verwendeter grammatischer Strukturen und idiomatischer Ausdrücke erkennen lasse, ob ein gebärdensprachliches Vorkommnis als authentisch wahrgenommen wird. Aus den genannten laienlinguistischen Einschätzungen wird ebenso deutlich, dass artikulatorischen Aspekten auf mimischer Ebene (siehe Kategorie III) eine entscheidende Rolle zugesprochen wird.

„Throughout the data, there is an overall consensus that the real story is told in and by a signer's face. In other words, when it comes to identifying articulatory features that mark the difference between sign language utterances that are perceived as authentic or inauthentic, specific importance is assigned to the face as articulator".

(Jaeger 2018: 83)

Im Bereich der relevanten mimischen Artikulation differenzieren die Probanden zwischen allgemeiner mimischer Aktivität (siehe IIIa) und dem, was auf dem Mund passiert (siehe IIIb). Zu den allgemeinen mimischen Elementen, welche als Authentizität indizierend von den Probanden genannt werden, gehören eine gewisse Lebendigkeit, welche in ihrer Intensität (stark vs. schwach), Quantität (viel vs. wenig) und Adäquatheit (entspricht der allgemeinen Gebärdengestalt vs. passt nicht) ausgewogen sein müssen (Jaeger 2018: 84). Im Hinblick auf artikulatorische Aspekte, welche am Mund produziert werden, waren sich die Probanden dahingehend einig, dass Mundgestiken als gebärdensprachimmanente Elemente (Sutton-Spence/Day 2000) eher authentische Sprachverwendung indizieren als Ablesewörter, welche Sprachkontaktphänomene zwischen Laut- und Gebärdensprachen darstellen (Ebbinghaus/Heßmann 1995) und ein DGS-Vorkommnis als nicht-authentisch erscheinen lassen. 
Die skizzierte Studie zeigt, dass Probanden auf meta-sprachlicher Ebene durchaus zwischen sprachlichen Realisierungen unterscheiden, welche aus ihrer Sich authentische oder nicht-authentische Sprachperformanz indizieren. Gleichzeitig fällt jedoch auch auf, dass, wenn es um die konkrete Benennung sprachlicher Merkmale geht, z. B. im Bereich mimischer Artikulationen, welche je nach Ausprägung aus laienlinguistischer Sicht mehr oder weniger mit Authentizität verknüpft werden, die von den Probanden offerierten Beschreibungen vage bleiben. Nichtsdestotrotz zeigt auch der Verweis auf eher schwammige Kategorien wie „Charisma“, dass ein Konzept wie ,,authentisches Gebärden“ selbst dann präsent sein kann, wenn dieses nicht in Worte gefasst werden kann. ${ }^{6}$

\subsection{TS_2: Authentizität - indiziert durch kreativen Sprachgebrauch}

In ihrer Studie untersuchen Jaeger und Harzer (2019), von welchen sprachlichen Mitteln gehörlose DGS-Nutzer Gebrauch machen, um sich selbst als authentische Gebärdensprachnutzer zu positionieren. Methodisch betrachten die Autorinnen das oben bereits skizzierte Datenmaterial auf zwei unterschiedlichen Ebenen. Als Ausgangspunkt für ihre Analysen wählen sie explizite, meta-sprachliche Kommentare der Teilnehmenden bezüglich ihrer Wahrnehmung, was authentisches Gebärden ausmacht. Die qualitative Analyse ihres Datenmaterials ergab, dass die Probanden im Rahmen der Fokusgruppen gemeinschaftlich etablieren, dass kreativer Sprachgebrauch essentielles Merkmal authentischer DGS-Verwendung ist. Daraufhin untersuchten Jaeger und Harzer (2019) in einem zweiten Schritt, inwiefern sich diese, auf meta-sprachlicher Ebene geäußerten Annahmen, in der de facto Sprachverwendung einzelner Gebärdensprachnutzer spiegelt.

Im Zuge der theoretischen Verortung ihrer Analyse, greifen Jaeger und Harzer (2019) auf Aghas (2003) Konzept der Registrierung (englisch: enregisterment) zurück. Im Sinne dieses Konzepts wird argumentiert, dass sprachliche Einheiten auf drei Ebenen registriert sein können. Die erste Ebene ist davon geprägt, dass bestimmte sprachliche Einheiten unmarkiert sind. I. e. bestimmte sprachliche Phänomene werden zwar verwendet und könnten folglich empirisch nachgewiesen werden. Für die Sprachverwender treten diese Elemente jedoch nicht in den Vordergrund. Im Kontrast dazu sind sich auf einer zweiten Registrierungsebene Sprachverwender der Benutzung bestimmter Elemente nicht nur sehr wohl bewusst, sondern auch in der Lage, sie in entsprechenden Situationen bewusst zu verwenden bzw. zu vermeiden. Ist ein sprachliches Phänomen auf dritter Ebene registriert, so erfüllt dieses eine indexikalische Funktion. I. e. unabhängig davon, ob ein sprachliches Merkmal de facto in der Sprachverwendung bestimmter Gruppen auftaucht, wird dieses Merkmal mit eben dieser Gruppe assoziiert.

Aufbauend auf diesem Konzept untersuchen Jaeger und Harzer (2019), inwiefern kreativer Sprachgebrauch in der DGS-Gemeinschaft authentischen Sprachgebrauch indiziert. Das von ihnen eingeführte Konzept „Creative Language Use (CLU)“ umfasst ein weites Spektrum an

\footnotetext{
${ }^{6}$ Fulford (1989: 7) argumentiert, dass man unterscheiden muss zwischen der Schwierigkeit ein Konzept zu verwenden und der Schwierigkeit dieses zu definieren. Verweisend auf das Konzept „Zeit“ erklärt er, dass dieses normalerweise problemlos verwendet wird. Gleichzeitig wäre es jedoch falsch anzunehmen, „that because conceptual difficulties are not normally raised by asking what time it is, the concept of "time" is transparent in meaning".
} 
artikulatorischen Möglichkeiten, welche im Hinblick auf inhaltliche Transparenz und kontextueller Gebundenheit variieren (Jaeger/Harzer 2019: 98).

Ungeachtet der Tatsache, dass die Grenzen zwischen bestimmten Formen kreativen sprachlichen Ausdrucks auf diesem Spektrum bei näherem Hinsehen verschwimmen, unterscheiden die Autorinnen zwischen den beiden Extremen sowie dem Bereich im Mittelfeld. An einem Ende des Spektrums lokalisieren sie jene Ausdrücke, welche sich auf der semantisch-pragmatischen Ebene lexikalisierter Ausdrücke bedienen. Das heißt, die semantische Bedeutung der verwendeten kreativen Gebärdenzeichen ist kontextuell ungebunden und somit transparent. An dem anderen Ende des Spektrums werden jene Ausdrücke verortet, welche sich bspw. durch spontane Gebärdenschöpfungen auszeichnen, die in höchstem Maße kontextuell gebunden sind. Dies betrifft sowohl ihre Produktion als auch ihre Rezeption (Jaeger/Harzer 2019: 98). Das heißt, man versteht die intendierte Bedeutung nur in der konkreten Situation.

Anhand der von ihnen analysierten Gesprächsdaten zeigen die Autorinnen auf, dass die Überzeugung ,,authentische Sprache ist kreative Sprache“ unter gehörlosen DGS-Nutzern in mehrfacher Hinsicht registriert ist. Das heißt, kreativer Sprachgebrauch wird auf meta-sprachlicher Ebene als Index für authentische DGS-Verwendung thematisiert, in seiner Verwendung als Instrument zur sozialen Selbstverortung als authentischer DGS-Nutzer im kommunikativen Raum eingesetzt und entsprechend von anderen Gesprächsteilnehmern interpretiert.

\subsection{TS_3: Der authentische Gebärdensprachnutzer}

Die von Jaeger (2019) präsentierten Ergebnisse beruhen auf der Auswertung von, im Kontext der oben bereits beschriebenen Fokusgruppen generierten, qualitativen Daten. In ihren Ausführungen beschäftigt sich die Autorin mit der Frage, wie die gehörlosen Probanden das Konzept des authentischen Gebärdensprachnutzers (the authentic signer, TAS) konstruieren.

Die Teilnehmenden wählen unterschiedliche Strategien der Fremd- vs. Selbstverortung als TAS. Ist bekannt - oder wird vermutet - dass das Gegenüber DGS als Erstsprache erworben hat, wird der Status TAS in erster Linie auf Basis dieses (vermeintlichen) Hintergrundwissens zugesprochen. Die de facto Sprachperformanz des Individuums wird zwar kommentiert, spielt im Zweifelfall jedoch eine untergeordnete Rolle. Wird also einer Person aufgrund ihrer Herkunft der Status TAS zugesprochen, wird es nicht als Index mangelnder Authentizität interpretiert, wenn entsprechende Erwartungen an das sprachliche Handeln nicht eingelöst werden. ${ }^{7} \mathrm{Im}$ Kontrast dazu erfolgt die Selbstverortung als TAS in erster Linie über den eigenen Sprachgebrauch. Die Teilnehmenden etablieren sich selbst als TAS, indem sie ausführen, dass sie sich bestimmte, Authentizität indizierende Mittel im Laufe der Zeit angeeignet haben und somit sie sich dieser zu bedienen wissen. Deutlich wird außerdem, dass die Verwendung Authentizität indizierender sprachlicher Merkmale insofern eine wesentliche Rolle spielt, als dass darüber soziales Prestige etabliert - oder einem Individuum zugesprochen - werden kann.

\footnotetext{
7 Ähnlich wie dies in dem von Matras (2015) genannten Beispiel beschrieben wurde, scheint auch hier die Annahme zu gelten: weil er oder sie in einem gehörlosen-gebärdensprachlichen Umfeld aufgewachsen ist, ist er oder sie authentisch und weil er oder sie authentisch ist, kann er oder sie authentisch gebärden, ungeachtet dessen, ob dies im vorliegenden Fall demonstriert wird.
}

ISSN 1615-3014 
Die Teilnehmenden unterscheiden in ihren Ausführungen zwischen Gebärdensprachnutzern, welche mit DGS als Erstsprache aufgewachsen sind und solche, welche DGS außerhalb des familiären Kontextes erworben haben. Da der aktive Gebärdensprachgebrauch bei der überwiegenden Mehrheit gehörloser Gebärdensprachnutzer nicht auf eine gebärdensprachliche Frühsozialisierung aufbaut, wird seitens der Teilnehmenden dargelegt, dass die Kompetenz „Authentizität" indizierende sprachliche Mittel zu verwenden das Ergebnis eines aktiven, zum Teil bewusst gesteuerten, Entwicklungsprozesses darstellt. Der Status TAS wird folglich, aus der Perspektive der Teilnehmenden betrachtet, als das Resultat eines Werdens-Prozesses konstruiert. Ungeachtet der Umstände, wie der Status TAS erworben wird, ist dieser stets explizit positiv konnotiert und mit sozialem Prestige assoziiert.

Zusammenfassend lässt sich festhalten, dass auf meta-sprachlicher Ebene ein gemeinschaftlich etabliertes Narrativ darüber existiert, durch welche sprachlichen Merkmale sich ,authentische Gebärdensprachverwendung“ auszeichnet. Diese ,authentische Gebärdensprachverwendung“ lässt sich auf metaphorischer Ebene als eine soziale Varietät der DGS konzeptualisieren (Jaeger 2018). Ebenso wurde gezeigt, dass auf pragmatischer Ebene die Verwendung von kreativem Sprachgebrauch als Index ,,authentischer Gebärdensprachverwendung“ registriert ist (Jaeger/Harzer 2019). Es wurde deutlich, dass die Verwendung Authentizität indizierender sprachlicher Mittel konkret mit der Person des authentischen Gebärdensprachnutzers verknüpft ist (Jaeger 2019).

\section{Diskussion}

Die oben rezipierten Studien zeigen, dass Authentizität indizierende sprachliche Mittel in der DGS normativen Charakter haben, wenn, wie zu Beginn des Aufsatzes dargelegt, sprachliche Normen im Spannungsfeld von Erwartung, Gebrauch und Bewertung verorten werden. In den drei rezipierten Teilstudien wird deutlich, dass Gebärdensprachnutzer eine klare Erwartungshaltung dahingehend haben, was sie unter authentischer Sprachverwendung verstehen. In der ersten Teilstudie (Jaeger 2018) wurde gezeigt, dass die Probanden auf der meta-Ebene über ein klares Konzept verfügen, wie authentisches Gebärden aussieht bzw. idealerweise auszusehen hat. Auf dieser Grundlage verfügen sie über eine Erwartungshaltung bezüglich dessen, was mit dem Label ,,authentische Gebärdensprachnutzung“" gekennzeichnet werden kann. Betrachtet man nun die in diesem Kontext aufgeführten sprachlichen und para-sprachlichen Mittel (u. a. Mimik, Lexikon, Charisma) unter normativen Aspekten, lässt sich argumentieren, dass sie einen Soll-Zustand skizzieren. Wenn also ,,authentische Gebärdensprachnutzung“ bestimmte linguistische und para-linguistische Kriterien aufweisen muss, um als solche erkannt zu werden, dann gewinnen diese sprachlichen Mittel auf der Basis einschlägiger Erwartungen normativen Charakter.

Während bei Jaeger (2018) der Aspekt der Erwartung im Vordergrund steht, gehen Jaeger und Harzer (2019) in der zweiten Teilstudie noch einen Schritt weiter. Sie zeigen, dass die Probanden nicht nur gemeinschaftlich einen Merkmalskatalog für ,authentische Gebärdensprachnutzung“ formulieren, sondern dass sich einzelne Personen in ihrer Sprachverwendung tatsächlich auch daran orientieren. So gesehen spiegelt sich die Erwartungshaltung, die von den Teilnehmenden im Hinblick auf ,,authentische Gebärdensprachverwendung“ formuliert wird, im konkreten Sprachgebrauch wider. Betrachtet man diesen Umstand aus normativer Perspektive, so 
lässt sich argumentieren, dass hier nicht nur eine Erwartungshaltung formuliert wird, sondern dass sich sprachliches Handeln tatsächlich auch daran orientiert; Das hier in den Vordergrund gerückte Merkmal kreativer Sprachgebrauch (creative language use, CLU) gewinnt normative Funktion, indem es als Index für ,,authentische Gebärdensprachverwendung“ erkannt und verwendet wird.

Die skizzierten sprachlichen Merkmale, welche aus Sicht von Gebärdensprachnutzern Authentizität indizieren, sind allesamt positiv konnotiert. Sie unterliegen einer Bewertung, welche insbesondere auf der meta-Ebene kommuniziert wird. Aus der dritten Teilstudie geht hervor, dass sich diese positive Bewertung genauso deutlich darin ausdrückt, dass der kompetente Einsatz genannter sprachlicher Mittel eng mit dem Status des authentischen Gebärdensprachnutzers (TAS) verknüpft wird (Jaeger 2019). Der Status TAS gilt als erstrebenswert, was sich u. a. darin zeigt, dass Teilnehmende ihre sprachliche Entwicklung als intentionalen Prozess beschreiben. Aus diesem Akt des Werdens lässt sich auch schließen, dass die Verwendung Authentizität indizierender sprachlicher Mittel mit einem gewissen Maß an gesellschaftlichem (i. e. innerhalb der DGS-Gemeinschaft) Prestige assoziiert wird.

Auf Grundlage der bisherigen Ausführungen lassen sich folgende Feststellungen treffen:

(1) In DGS gibt es gebärdensprachliche und para-sprachliche Mittel, welche mit authentischen Gebärdensprachgebrauch assoziiert werden.

(2) Auf Grundlage des gemeinschaftlich etablierten Narrativ lässt sich schließen, dass hinsichtlich der gebärdensprachlichen Realisierung dieser Mittel ein kollektives Normwissen existiert.

(3) Dass dieses Normwissen als handlungsleitend akzeptiert wird, lässt sich daran erkennen, dass individuelle Gebärdensprachnutzer sich ganz bewusst entsprechende sprachliche Mittel aneignen und diese gezielt einsetzen, um sich als authentische Gebärdensprachverwender sozial zu positionieren.

(4) Bezugnehmend auf ihren handlungsleitenden Charakter lassen sich die Authentizität indizierenden gebärdensprachlichen Mittel als Authentizitätsnormen konzeptualisieren.

In diesem Zusammenhang eröffnet die von Gloy (1993) vorgeschlagene Differenzierung zwischen Standard und Hochsprache nicht nur neue Perspektiven, sondern bietet den notwendigen theoretischen Rahmen, um die präsentierten Ergebnisse sinnvoll zu verorten. Geht man davon aus, dass es eine soziale Varietät innerhalb der DGS gibt, welche sich einerseits insbesondere durch Authentizität indizierende sprachliche und para-sprachliche Merkmale auszeichnet, und andererseits positiv konnotiert (und somit prestigeträchtig) ist, so lässt sich mutmaßen, dass es sich hierbei um eine Ausprägung von Standard-DGS handeln könnte. Betrachtet man diverse Ausführungen zum Thema „Standardvarietäten“ im Zusammenhang mit Lautsprachen, wird jedoch schnell deutlich, dass der Faktor ,soziales Prestige“, neben Aspekten wie supraregionale Verbreitung, situativer Verortung (i. e. bevorzugte Anwendung in formellen Kontexten) und medialer Realisierung (besonders häufiges Auftreten in schriftlichen Texten), durchaus zwar auch eine, jedoch nicht zwangsweise die wichtigste, Rolle spielt. Während die genannten gebärdensprachlichen Merkmale eine möglicherweise existierende soziale Varietät ,authentische-DGS“ auszeichnen, bleibt unklar, inwiefern andere Kriterien, die in lautsprachbezogenen Diskussionen als für eine Standardvarietät relevant erachtet werden, auf diese ebenso zutreffen. 
Insofern ist es zum jetzigen Zeitpunkt verfrüht zu postulieren, ,authentische DGS“ sei mit „Standard DGS“ gleichzusetzen.

Im Gegensatz dazu zeigen die rezipierten Studien gleichzeitig jedoch, dass sprachliche Realisierungen, welche mit ,authentischer-DGS“ assoziiert werden, durchaus Hochsprachlichkeit im Gloy'schen Sinne darstellen, und somit normativen Charakter gewinnen. I. e. die Verwendung Authentizität indizierender sprachlicher Mittel kennzeichnet ,eine Prestige-Varietät, deren Beschaffenheit und Wert sich nicht an Kodifikationen, auch nicht an Überregionalität bemißt, sondern allein daran, wer diese Varietät spricht“ (Gloy 1993: 55). „Wer“ verweist in diesem Zusammenhang auf ,authentische Gebärdensprachnutzer“, welche sich u. a. durch die Verwendung Authentizität indizierender gebärdensprachlicher Mittel sozial positionieren.

Zurückgreifend auf die zu Beginn des Aufsatzes theoretischen Auseinandersetzungen mit Authentizität ist anzumerken, dass die Verknüpfung zwischen bestimmten sprachlichen und parasprachlichen Merkmalen, ,authentischer DGS“ und dem ,authentischen Gebärdensprachverwender" (TAS) essentialistische Perspektiven auf Authentizität nahelegt. Dies ist zwar nicht mit konstruktivistischen Positionen vereinbar (Eckert 2003; Coupland 2003; Bucholtz 2003) und somit im Kontext aktueller soziolinguistischer Diskurse kritisch zu hinterfragen, reflektiert jedoch gleichzeitig eine Perspektive, welche aus Sicht der Teilnehmenden stimmig zu sein scheint. Die Konstruktion des TAS - insbesondere im Hinblick auf die Selbstverortung als TAS - zeigt jedoch auch, dass sprachliche Authentizität nicht zwangsläufig etwas Statisches ist, sondern von den Teilnehmenden auch als dynamisches Konstrukt verstanden wird, welches kontextuell aktiv ausgehandelt werden kann.

\section{$5 \quad$ Zusammenfassung und Schlussfolgerungen}

Die vorangegangenen Überlegungen verdeutlichen die Übertragbarkeit von Konzepten aus der Lautsprach- in die Gebärdensprachforschung. Dadurch ergibt sich eine Perspektivenerweiterung, die Aussicht auf neue Erkenntnisse eröffnet. Zunächst wurde anhand empirischer Daten aufgezeigt, dass sich in der DGS-Gemeinschaft Authentizitäts-Merkmale identifizieren lassen, welche sowohl sprachlich realisiert, als auch auf metasprachlicher Ebene registriert sind. Diese lassen sich als Ausprägung von Authentizitätsnormen konzeptualisieren und entsprechend theoretisch einordnen. Werden Authentizitätsnormen nicht bedient, so hat dies Konsequenzen für den individuellen Sprachnutzer. Das positiv konnotierte Attribut ,authentisch“ wird ihm/ihr nicht zugeschrieben. Es ist davon auszugehen, dass denjenigen, welche sich in ihrem Sprachgebrauch an Authentizitätsnormen orientieren, eine sprachliche Vorbildfunktion und somit soziales Prestige zugesprochen wird. In welchem Ausmaß sich so eine positive Sanktionierbarkeit ausdrückt, ist eine Frage, die es in zukünftigen Studien zu untersuchen gilt.

Darüber hinaus wurde am Beispiel der Sprachverwendung in der DGS-Gemeinschaft gezeigt, dass die von Gloy (1993) vorgeschlagene Unterscheidung zwischen Standard und Hochsprache konzeptuell erfasst und terminologisch explizit macht, was bis heute in der Literatur noch viel zu selten ausbuchstabiert wird. Es konnte gezeigt werden, dass es in der DGS-Gemeinschaft hochsprachliche Ausprägungen gibt, welche jedoch nicht mit herkömmlichen Definitionen von Standardvarietät(en) zu erfassen sind. Die Ausführungen legen nahe, dass der Terminus „Hochsprachlichkeit" keineswegs lediglich nur eine Seite einer zwei-seitigen Standard-HochspracheMedaille in den Vordergrund rückt. Während Gloy (1993) noch betont, dass es wichtig ist, die 
linguistische und soziale Seite einer sprachlichen Varietät explizit zu fokussieren, sprechen die hier betrachteten Untersuchungen dafür, Hochsprache und Standardsprache als zwei unterschiedliche sprachliche Ausprägungen zu konzeptualisieren, welche zwar in ihrer Ausführungsform identisch sein können, es aber keineswegs sein müssen.

\section{Literaturverzeichnis}

Adam, Robert (2015): “Standardization of Sign Languages". Sign Language Studies 15/4: 432-45.

Agha, Asif (2003): The social life of cultural value. Language and Communication 23, 3-4: 231-273. doi:10.1016/S0271-5309(03)00012-0.

Akande, Akinmade T. (2014): "Hybridity as authentic in Nigerian hip-hop lyrics". In: Lacoste, Véronique/Leimgruber, Jakob/Breyer, Thiemo (eds.): Indexing Authenticity: Sociolinguistic Perspectives. Berlin/New York, de Gruyter: 269-286.

Ammon, Ulrich (2003): On the Social Forces that Determine what is Standard in a Language. Sociolinguistica 17. 1-10.

Ammon, Ulrich (2005): „Standard und Variation: Norm, Autorität, Legitimation“. In: Eichinger, Ludwig M./Kallmeyer, Werner (eds.): Standardvariation. Wie viel Variation verträgt die deutsche Sprache? Berlin/New York, de Gruyter: 28-40.

Berend, Nina (2005): Regionale Gebrauchsstandards - Gibt es sie und wie kann man sie beschreiben? In: Eichinger, Ludwig M./Kallmeyer, Werner (eds.): Standardvariation. Wie viel Variation verträgt die deutsche Sprache? Berlin/New York, de Gruyter: 143-170.

Bex, Tony/Watts, Richard J. (1999): Standard English: The Widening Debate. London: Routledge.

Brommer, Sarah (2015): „Sprachliche Muster als Indikator für die Angemessenheit eines Textes - Grundlagen einer automatisierten Text- und Stilanalyse“. Aptum. Zeitschrift für Sprachkritik und Sprachkultur 11/2: 121-130.

Bucholtz, Mary (2003): Sociolinguistic nostalgia and the authentication of identity. Journal of Sociolinguistics 7/3: 398-416. doi:10.1111/1467-9481.00232.

Buendgens-Kosten, Judith (2014): „Authenticity“. ELT Journal 68/4: 457-459.

Buendgens-Kosten, Judith. 2013: "Authenticity in CALL: Three domains of "realness"”. ReCALL 25/2: 272-285.

Bußmann, Hadumod (2008. Lexikon der Sprachwissenschaft. Stuttgart: Alfred Kröner Verlag.

Coupland, Nikolas (2003): "Sociolinguistic authenticities". Journal of Sociolinguistics 7/3: 417-431. doi:10.1111/1467-9481.00233.

Coupland, Nikolas (2007): Style: Language Variation and Identity. Cambridge: Cambridge University Press.

Coupland, Nikolas (2014): "Language, society and authenticity: Themes and perspectives". In: Lacoste, Véronique/Leimgruber, Jakob/Breyer, Thiemo (eds.): Indexing Authenticity: Sociolinguistic Perspectives. Berlin, de Gruyter: 14-39.

Coupland, Nikolas (2007): Style: Language Variation and Identity. Cambridge: Cambridge University Press.

Dittmar, Norbert. 1997. Grundlagen der Soziolinguistik - Ein Arbeitsbuch mit Aufgaben. Tübingen: Niemeyer.

Ebbinghaus, Horst/Heßmann, Jens (1995): „Formen und Funktionen von Ablesewörtern in 
gebärdensprachlichen Äußerungen (Teil 2)“. Das Zeichen 31: 50-61.

Eckert, Penelope (2003): "Sociolinguistics and authenticity: An elephant in the room". Journal of Sociolinguistics 7/3: 392-397.

Eichinger, Ludwig M. (2005): Standardnorm, Sprachkultur und die Veränderung der normativen Erwartungen. In: Eichinger, Ludwig M./Kallmeyer, Werner (eds.): Standardvariation: Wie viel Variation verträgt die deutsche Sprache? Berlin/New York, de Gruyter: 363-381. doi.org/10.1515/9783110193985.363.

Eichmann, Hanna (2009a): „Gebärdensprachstandardisierung: ein Konzept wird hinterfragt“. Das Zeichen 23/82: 292-306.

Eichmann, Hanna (2009b): "Planning sign languages: promoting hearing hegemony? Conceptualizing sign language standardization”. Current Issues in Language Planning 10/3: 293-307.

Eichmann, Hanna (2013): "Hands off our language!" Deaf sign language teachers' perspectives on sign language standardisation. Aachen: Shaker.

Fix, Ulla (1995): „Textmusterwissen und Kenntnis von Kommunikationsmaximen. Voraussetzung, Gegenstand und Ziel der kommunikationsbezogenen Sprachberatung“. In: Biere, Bernd Ulrich/Hoberg, Rudolf (eds.): Bewertungskriterien in der Sprachberatung. Tübingen, Narr: 113-144.

Fulford, Kenneth Willliam Musgrave. 1989. Moral theory and medical practice. Cambridge: Cambridge University Press.

Gadamer, Hans-Georg (1960): Wahrheit und Methode. Tübingen: Mohr.

Gilmore, Alex (2007): "Authentic materials and authenticity in foreign language learning". Language Teaching 40/02: 97-118.

Gloy, Klaus (1975): Sprachnormen I. Linguistische und soziologische Analysen. Stuttgart Bad Cannstadt: Frommann.

Gloy, Klaus (1993): „Sprachnormenforschung in der Sackgasse? Überlegungen zu Renate Bartsch, Sprachnormen: Theorie und Praxis“. Beiträge zur Geschichte der deutschen Sprache und Literatur 115: 30-65.

Gloy, Klaus (1995): Zur Methodologie der Sprachnormen-Forschung. Rostocker Beiträge zur Sprachwissenschaft 1.73-93.

Gloy, Klaus (2004): „Norm/Norm“. In: Ammon, Ulrich/Dittmar, Norbert/Mattheier, Klaus J. (eds.): Soziolinguistik. 2. Bd. Berlin/New York: de Gruyter. (= HSK 3.2).

Hartung, Wolfdietrich (1977): „Zum Inhalt des Normbegriffs in der Linguistik.“ In: Hartung, Wolfdietrich (ed.): Normen in der sprachlichen Kommunikation. Berlin, Akademie-Verlag: 9-69.

Heidegger, Martin (1927): Sein und Zeit. Tübingen: Niemeyer.

Jaeger, Hanna/Harzer, Sarah (2019): “'Authentic signing equals creative signing': New perspectives on the enregisterment of creative language use in German Sign Language". Lingua. 217: 90-100. doi:10.1016/j.lingua.2018.10.013.

Jaeger, Hanna (2017): Modality matters: On social forces determining what is standard in German Sign Language (DGS). Linguistik Online 81(2). 45-58.

Jaeger, Hanna (2018): Mapping (in)authenticity: Folk accounts of sociolinguistic variation in German Sign Language. Sign Language Studies 19(1). 75-99.

Jaeger, Hanna (2019): “'Not like them': New Signers ' narrative constructions of the authentic 
signer". Ampersand 6: 100048. doi:10.1016/j.amper.2019.100048.

Johnston, Trevor (2003): "Language standardization and sign language dictionaries." Sign Language Studies 3/4: 431-468.

Krentz, Christoph (2006): "The camera as printing press". In: Bauman, H-Dirksen L./Nelson, Jennifer L./Rose,Heidi M. (eds.): Signing the Body Poetic: Essays on American Sign Language Literature. Berkeley, University of California Press: 51-70.

Labov, William (1972): Sociolinguistic patterns. Philadelphia: University of Pennsylvania Press.

Lacoste, Véronique/Leimgruber, Jakob/Breyer, Thiemo (2014):" Authenticity: a view from inside and outside sociolinguistics". In: Lacoste, Véronique/Leimgruber, Jakob/Breyer, Thiemo (eds.): Indexing Authenticity: Sociolinguistic Perspectives. Berlin/New York, de Gruyter: 1-13.

Lange, Maria Barbara (2008): Sprachnormen im Spannungsfeld schriftsprachlicher Theorie und Praxis. Berlin: de Gruyter.

Langer, Gabriele et al. (2018): "Authentic Examples in a Corpus-Based Sign Language Dictionary - Why and How". In: Čibej, Jaka et al. (eds.): Proceedings of the XVIII EURALEX International Congress: Lexicography in Global Contexts. Ljubljana, Ljubljana University Press, Faculty of Arts: 483-497.

Löffler, Heinrich (2005): „Wieviel Variation verträgt die deutsche Standardsprache? Begriffsklärung: Standard und Gegenbegriffe“. In: Eichinger, Ludwig M./Kallmeyer, Werner (eds.): Standardvariation. Wie viel Variation verträgt die deutsche Sprache? Berlin/New York, de Gruyter: 7-27.

Lowe, Robert J./Pinner, Richard (2016. Finding the connections between native-speakerism and authenticity. Applied Linguistics Review 7/1: 27-52. doi:10.1515/applirev-2016-0002.

Matras, Yaron (2015): “Transnational policy and 'authenticity' discourses on Romani language and identity". Language in Society 44/03: 295-316.

Mitchell, Ross E./Michael Karchmer (2004): "Chasing the Mythical Ten Percent: Parental Hearing Status of Deaf and Hard of Hearing Students in the United States". Sign Language Studies 4/2: 138-163.

Morford, Jill P./Nicodemus, Brenda/Wilkinson, Erin (2015): "Research Methods in Psycholinguistic Investigations of Sign Language Procesing”. In: Orfanidou, Eleni/Woll, Bencie/Morgan, Gary (eds.): Research Methods in Sign Language Studies: A Practical Guide. Oxford: Wiley Blackwell: 209-249.

Napier, Jemina/Leeson, Gary (2016): Sign Language in Action. London: Palgrave Macmillan UK.

Niedzielski, Nancy/Preston, Dennis R. (2000): Folk linguistics. Berlin/New York: de Gruyter.

O'Rourke, Bernadette/Ramallo, Fernando (2013): "Competing ideologies of linguistic authority amongst new speakers in contemporary Galicia". Language in Society 42/03: 287-305.

Pearce, Michael. 2012. Folk accounts of dialect differences in Tyne and Wear. DiG 20. 5-25. Pietikäinen, Sari et al. (2016): Sociolinguistics from the Periphery. Small Languages in new Circumstances. Cambridge: Cambridge University Press.

Richter, Dagmar (2005): Sprachenordnung und Minderheitenschutz im schweizerischen Bundesstaat. Relativität des Sprachenrechts und Sicherung des Sprachfriedens. 
Berlin: Springer.

Rose, Tricia (2008): The Hip Hop Wars. New York: Basic Civitas Books Publishers.

Sartre, Jean-Paul (1945): L'être et le néant. Essai d'ontologie phénoménologique. Paris: Gallimard.

Schermer, Trude (2012): “Sign Language Planning in the Netherlands between 1980 and 2010". Sign Language Studies 12/4. 467-493. doi:10.1353/sls.2012.0016.

Schmitt, Christian (2001): „Sprachnormierung und Standardsprachen.“ In: Holtus, Günter/Metzeltin, Michael/Schmitt, Christian (eds.): Lexikon der Romanistischen Linguistik (LRL). Vol. I, 2: Methodologie (Sprache in der Gesellschaft/Sprache und Klassifikation/Datensammlung und -verarbeitung). Méthodologie (Langue et sociétél Langue et classification/Collection et traitement des do, 435-492. Tübingen: Niemeyer.

Schneider, Jan Georg/Albert, Georg (2013): „Medialität und Standardsprache - oder: Warum die Rede von einem gesprochenen Gebrauchsstandard sinnvoll ist“". In: Hagemann, Jörg/ Klein, Wolf Peter/Staffeldt, Sven (eds.): Pragmatischer Standard. Tübingen, Stauffenburg: 49-60.

Senatsverwaltung für Bildung, Jugend und Familie (Berlin) und Ministerium für Bildung, Jugend und Sport Land Brandenburg (2015): Rahmenlehrplan Teil C: Deutsche Gebärdensprache, Jahrgangsstufen 1-10. https://bildungsserver.berlin-brandenburg.de/. https://bildungsserver.berlin-brandenburg.de/fileadmin/bbb/unterricht/rahmenlehrplaene/ Rahmenlehrplanprojekt/amtliche_Fassung/Teil_C_DGS_2015_11_16_WEB.pdf. [25.11.2019]

Spitzmüller, Jürgen (2013): Graphische Variation als soziale Praxis. Eine soziolinguistische Theorie skripturaler ,Sichtbarkeit'. Berlin: de Gruyter. doi:10.1515/zrs-2014-0010.

Sutton-Spence, Rachel/Day, Linda (2000): "Mouthings and mouth gestures in British Sign Language (BSL)". In: Boyes Braem, Penny/Sutton-Spence, Rachel (eds.): The hands are the head of the mouth: The mouth as articulator in sign languages. Hamburg, Signum: 69-85.

Tauber, Walter (1993): Mundart und Schriftsprache in Bayern (1450-1800). Berlin/New York: de Gruyter.

Techtmeier, Bärbel (1977): „Die kommunikative Adäquatheit sprachlicher Äußerungen“. Normen in der sprachlichen Kommunikation. Berlin: Akademie Verlag: 102-162.

Woll, Bencie (2013): „Sign language and spoken language development in young children: Measurung vocabulary by means of the CDI". In: Meurant, Laurence et al. (eds.): Sign Language Research, Uses and Practices. Berlin/Boston/Nijmegen: de Gruyter Mouton and Ishara Press: 15-34. 